\title{
Evaluation of Patient-Reported Outcomes and Quality of Life with the I-neb AAD System in Patients with Chronic Obstructive Pulmonary Disease
}

\author{
Nicola Goodman, B.Sc., Mike Morgan, M.D., FRCP,' Kurt Nikander, B.A., ${ }^{1}$ \\ Sarah Hinch, B.Sc., ${ }^{3}$ and Steven Coughlin, Ph.D. ${ }^{3}$
}

\begin{abstract}
Background: The I-neb Adaptive Aerosol Delivery (AAD) System is a novel portable mesh nebulizer that provides patient feedback regarding adherence to prescribed treatment and compliance with the correct use of the device.

Methods: This multicenter study was composed of 98 patients aged 53-80 years with Chronic Obstructive Pulmonary Disease (COPD). The primary variables were ease of use and satisfaction, which were assessed after 3 months of use of the I-neb AAD System (assessed at visit 2) and after 3 months of use of the patient's previous nebulizer system (assessed at visit 1) using matched questions from pre- and poststudy questionnaires. Quality of life was also assessed at visits 1 and 2 using the validated Chronic Respiratory Questionnaire (CRQ), which consists of dyspnea, emotional function, fatigue, and mastery domains. Differences in the distribution of responses between the pre- and poststudy ease of use and satisfaction questions were analyzed using the Marginal Homogeneity test. Differences in mean CRQ scores between the pre- and poststudy assessments were analyzed using the Wilcoxon Signed-Rank test.

Results: Patient responses on the ease of use and satisfaction questions significantly $(p \leq 0.001)$ favored the I-neb AAD System compared with the patient's previous nebulizer system. In addition, significant $(p \leq 0.015)$ improvements in the CRQ dimensions of dyspnea and fatigue were reported with the I-neb AAD System compared with the patients' previous nebulizer systems.

Conclusions: The results from this study demonstrated that patients were more satisfied with the I-neb AAD System and found it easier to use than their previous nebulizer systems. In addition, the I-neb AAD System significantly improved dyspnea and fatigue compared with the patients' previous nebulizer systems, which may reflect improved adherence or more correct use of the nebulizer system with the I-neb AAD System.
\end{abstract}

Key words: I-neb AAD System, Adaptive Aerosol Delivery, ease of use, patient satisfaction, quality of life, nebulizer, mesh nebulizer

\section{Introduction}

$\mathbf{I}_{\mathrm{f} w \mathrm{~m}}^{\mathrm{N}}$ NHALED BRONCHODILATORS are the mainstay of treatment for Chronic Obstructive Pulmonary Disease (COPD) for control and prevention of symptoms. ${ }^{(1)}$ Inhaled corticosteroids (ICSs) are used to decrease the frequency of exacerbations, particularly in patients with advanced COPD with a history of repeat exacerbations. ${ }^{(1,2)}$ Selection of the most ap- propriate aerosol delivery system for inhaled COPD therapies requires consideration of the patient's ability to correctly use the device, patient preference, convenience (e.g., portability), availability of the device for use with all of the patient's medications, and treatment time. ${ }^{(3,4)}$

Nebulizers are the accepted inhalation device of choice for patients unable to coordinate inhalation and actuation of a pressurized metered dose inhaler ( $\mathrm{pMDI}$ ) or to provide the

${ }^{1}$ Glenfield Hospital, Leicester, United Kingdom.

${ }^{2}$ Philips Respironics, Respiratory Drug Delivery, Parsippany, New Jersey.

${ }^{3}$ Philips Respironics, Respiratory Drug Delivery (UK) Ltd, Chichester, United Kingdom. 
inspiratory flow necessary for effective use of a dry powder inhaler (DPI). ${ }^{(3)}$ Three classes of nebulizer exist: jet, ultrasonic, and mesh. ${ }^{(5)}$ Although jet nebulizer-compressor systems are used commonly, they require a power source and are often bulky. ${ }^{(3)}$ Conventional ultrasonic nebulizers are more compact but cannot be used to deliver proteins ${ }^{(6)}$ or suspensions. ${ }^{(7,8)}$ Because vibrating mesh nebulizers use lower frequency waves than traditional ultrasonic nebulizers, heating issues that denature proteins are avoided. $(6,9)$ Mesh nebulizers deliver medications by forcing liquid through multiple apertures in a vibrating alloy-metal mesh plate. ${ }^{(10,11)}$

The I-neb Adaptive Aerosol Delivery (AAD) System (Philips Respironics, Respiratory Drug Delivery (UK) Ltd, Chichester, UK) (Fig. 1) is a novel mesh nebulizer that is portable $(150 \times 65 \times 45 \mathrm{~mm}, \mathrm{~h}, \mathrm{w}, \mathrm{d})$, lightweight $(210 \mathrm{~g})$, and virtually silent. ${ }^{(10)}$ In Europe, the I-neb AAD System is approved as a small volume nebulizer. ${ }^{(10)}$ In the United States, the I-neb AAD System is approved only for use with specific medications. ${ }^{(10,11)}$ The AAD technology detects changes in the patient's breathing pattern and adjusts to deliver aerosolized medications during inhalation only. ${ }^{(10)}$ Additionally, the I-neb AAD System has been designed with the capability to record adherence to prescribed treatment regimen and compliance with the correct use of the device via a built in patient logging system (PLS). ${ }^{(10)}$ A device that enables correct patient use and facilitates adherence may be beneficial as poor adherence to treatment and compliance with the correct use of the nebulizer is common in patients with COPD. ${ }^{(12,13)}$

As part of a 3-month study in the United Kingdom evaluating patient handling and reliability of the I-neb AAD System in a usual care setting, we assessed patient-reported outcomes. We compared patient satisfaction, device ease of use, and patient quality of life on inhaled COPD therapy delivered via the I-neb AAD System versus the patients' previous nebulizer systems.

\section{Materials and Methods}

\section{Patients}

Patients aged 50-80 years with COPD diagnosed according to the National Institute for Health and Clinical Excellence guidelines were eligible for this study. ${ }^{(14)}$ In addition, patients were required to have the ability to take their bronchodilator and/or corticosteroid medications through a standard nebulizer with a mouthpiece. Patients were excluded if they were on continuous oxygen therapy, had clinically significant exacerbation of respiratory disease in the previous 4 weeks, or had participated in any other clinical trial in the previous 4 weeks. The patients were recruited from a database of domiciliary nebulizer users. They had nebulizers supplied after a formal assessment that objectively demonstrated that they would benefit from the use of a nebulizer.

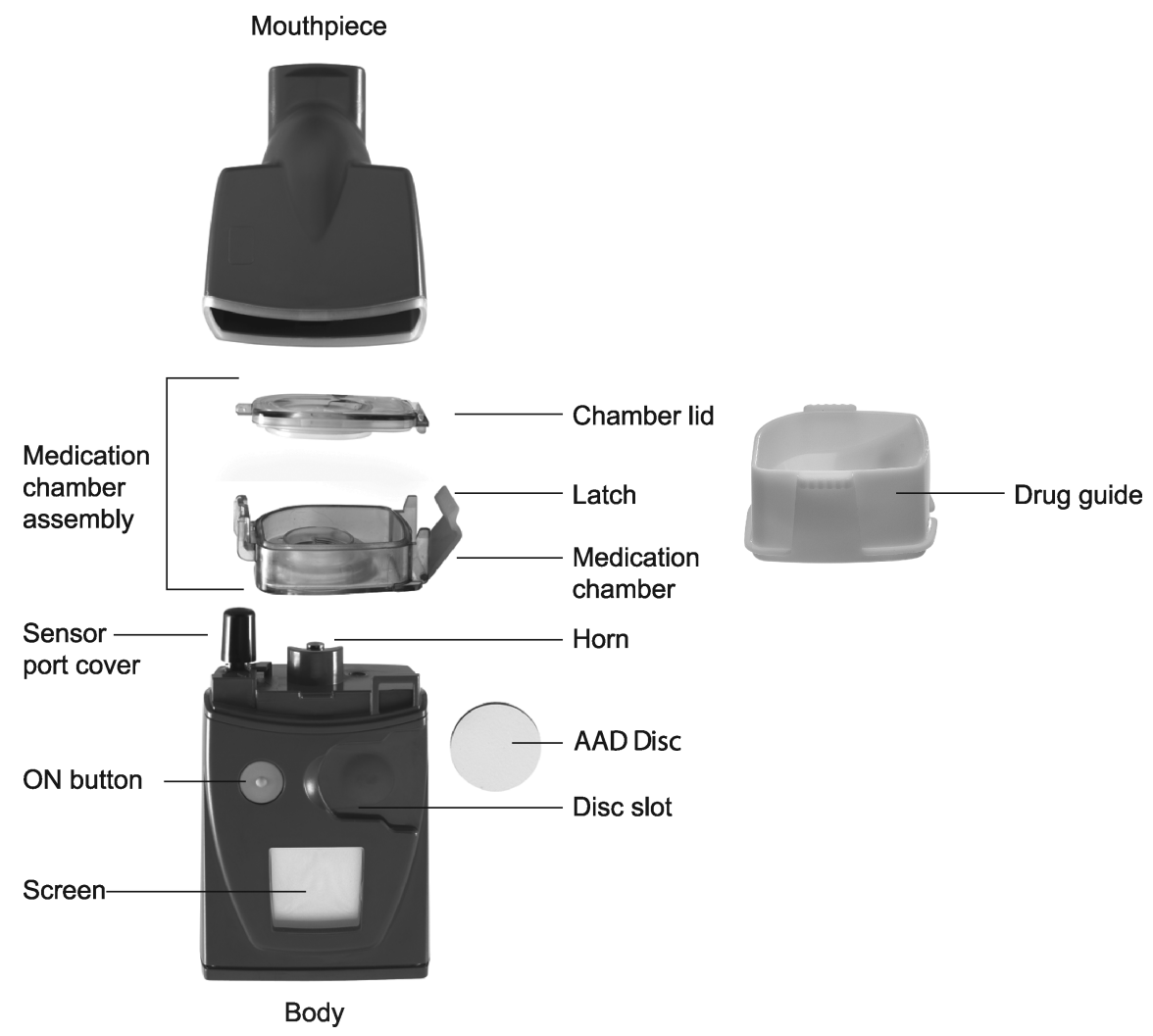

FIG. 1. The I-neb Adaptive Aerosol Delivery (AAD) System. The main components of the device are the mouthpiece, the medication chamber assembly, and the body. The I-neb AAD System has been designed to deliver aerosol with two different breathing pattern algorithms, the Tidal Breathing Mode (TBM) and the Target Inhalation Mode (TIM). In TIM the inspiratory flow through the valve in the mouthpiece is limited to $\sim 20 \mathrm{~L} / \mathrm{min}$. 


\section{Study design}

This multicenter study consisted of two clinic visits (one pre- and one posttreatment) separated by a 3-month treatment period. During visit 1, patients completed prestudy questionnaires that assessed ease of use, patient satisfaction, and quality of life during the previous 3 months of treatment with their usual nebulizer device. During visit 1, patients were also trained on the correct use of the I-neb AAD System and were instructed to use it only for administration of their prescribed bronchodilator and/or corticosteroid medications during the 3-month study period; other inhaled medications were to be administered using their usual nebulizer device. During visit 2, patients completed poststudy questionnaires assessing ease of use, patient satisfaction, and quality of life during the 3 months of treatment with the I-neb AAD System. Compliance with the correct use of the I-neb AAD System was assessed based on data downloaded from the PLS component of the I-neb AAD System.

The study protocol was approved by the Leicestershire, Northamptonshire \& Rutland Local Research Ethics Committee Two, and the study was conducted in accordance with ethical principles based on the Declaration of Helsinki. Written informed consent was obtained from patients before they entered the study.

\section{Outcome measures}

The primary variables were ease of use of and patient satisfaction with the I-neb AAD System compared with the patient's previous nebulizer system after 3 months of use of each. The questions related to ease of use and patient satisfaction were developed specifically for this study and were not formally validated. Ease of use was assessed based on patient responses to five matched questions from the preand poststudy device usage questionnaires; patient satisfaction was assessed based on patient responses to four matched questions from the pre- and poststudy device usage questionnaires. Responses for ease of use and satisfaction were scored on a 5-point Likert scale. The poststudy device usage questionnaire included four additional end-of-study satisfaction questions that asked patients to compare the I-neb AAD System with their previous nebulizer.

Quality of life during treatment with the I-neb AAD System compared with during treatment with the patients' previous nebulizer systems was assessed as a secondary variable using the validated Chronic Respiratory Questionnaire (CRQ). ${ }^{(15)}$ The CRQ comprised four domains relating to dyspnea, emotional function, fatigue, and mastery, all scored on a 7-point scale, with lower scores indicating a greater level of dysfunction. The CRQ dimensions were standardized except for dyspnea, which required the patients to identify the five most important everyday activities that made them breathless and then score these activities on the same 7-point scale. Adverse events were also recorded.

\section{Statistical analyses}

Analyses were based on the per-protocol population. Differences in the distribution of responses between the pre- and poststudy ease of use and satisfaction questions were analyzed using a Marginal Homogeneity test (Pearson's $\chi^{2}$ test).
Patient responses to each question were included in the analysis only if pre- and poststudy responses existed for the same question. Differences in mean CRQ scores between the pre- and poststudy CRQ assessments were analyzed using the Wilcoxon Signed-Rank test. A difference of $\geq 0.5$ on each CRQ dimension was considered clinically meaningful. ${ }^{(16)}$ It was determined that a sample size of 100 patients would allow for estimation of the proportion of patients reporting responses of "easy" or "very easy" on the ease of use questions or "like" or "really like" on the satisfaction questions to within 0.09 of the expected proportion of 0.70 with a two-sided 95\% confidence interval of 0.61-0.79.

\section{Results \\ Patients}

Ninety-eight patients were screened and enrolled in the study; 28 patients were excluded from the per-protocol analysis because of study withdrawal $(n=27)$ or lack of data regarding inclusion and exclusion criteria $(n=1)$. Of the 70 patients included in the per-protocol population, the median age was 68 years (range: $53-80$ ) and $51 \%$ were male. Before the start of the study, approximately $96 \%$ of the patients used a jet nebulizer to deliver their inhaled medication and $4 \%$ used an ultrasonic nebulizer. At visit 1, most patients were using their current nebulizer system for delivery of bronchodilators $(99 \%)$ or corticosteroids $(4 \%)$; only $3 \%$ of patients were using their current nebulizer system for delivery of medications other than bronchodilators or corticosteroids. Most patients used their current nebulizer system either one to two times per day (41\%) or three to four times per day (58\%). Data downloaded from the PLS function indicated that $95.1 \%$ of the doses that were delivered from the I-neb AAD System during the 3-month study period were administered at the correct dose.

\section{Ease of use}

The distribution of patient responses to the matched preand poststudy ease of use questions significantly favored the I-neb AAD System over the patients' previous nebulizer systems for four out of five questions: ease of assembly $(p=0.0001)$, ease of taking apart $(p<0.0001)$, ease of cleaning $(p<0.0001)$, and confidence that drug was delivered into the lungs ( $p=0.001$ ) (Table 1 and Fig. 2A and B). The distribution of patient responses for confidence in using the nebulizer system correctly was not significantly different between the pre- and poststudy questions.

\section{Patient satisfaction}

The distribution of patient responses to the matched preand poststudy satisfaction questions significantly favored the I-neb AAD System compared to the patients' previous nebulizer systems for all the satisfaction parameters assessed, including portability, shape, size, and weight of the nebulizer $(p<0.0001)$ (Table 2 and Fig. 3). Similarly, the distribution of patient responses to the comparative satisfaction questions at the end of the study favored the I-neb AAD System over the patients' previous nebulizer systems for all parameters assessed, including ease of operation, ease of fitting into the usual routine, confidence that drugs were getting delivered 


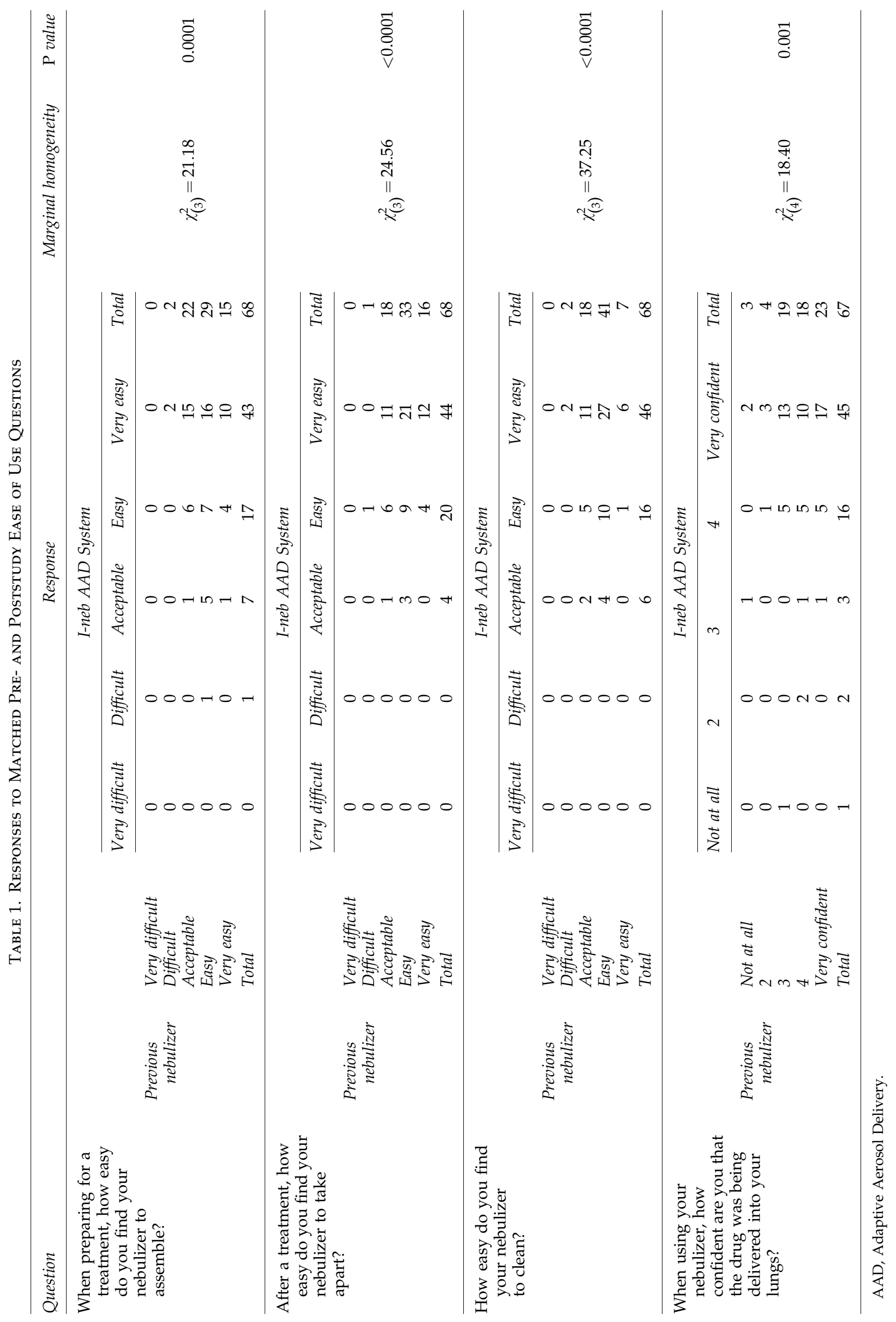


A

Ease of assembly $(n=68)$

Previous nebulizer

I-neb AAD System

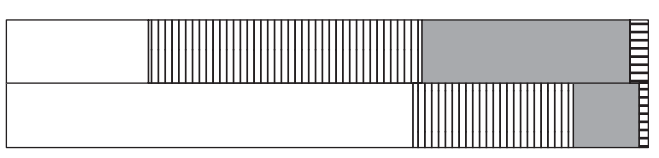

Ease of taking apart $(n=68)$

Previous nebulizer

I-neb AAD System

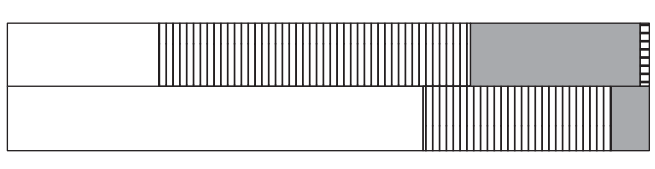

$\square$ Very easy

m. Easy

$\square$ Acceptable

目Difficult

- Very difficult

Ease of cleaning $(n=68)$

Previous nebulizer

I-neb AAD System
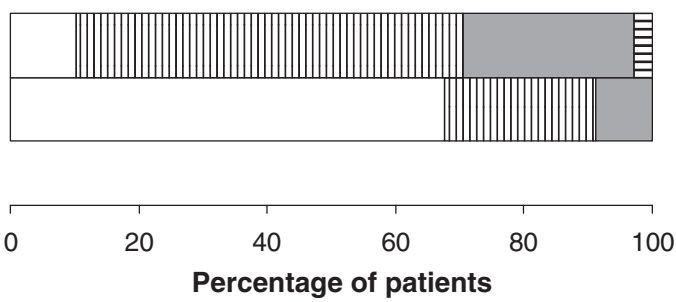

B

Confidence that drug was

delivered into lungs $(n=67)$

Previous nebulizer

I-neb AAD System

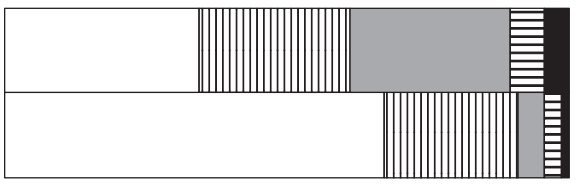

$\square$ Very confident

$\mathbb{1} 4$

$\square 3$

E2

Confidence in using

not very confident

nebulizer correctly $(n=66)$

Previous nebulizer

I-neb AAD System
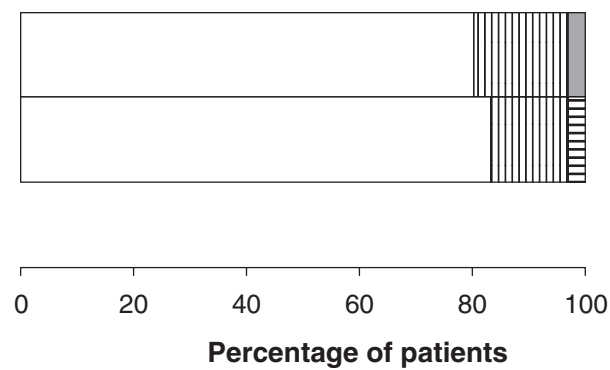

FIG. 2. Percentage of patients reporting each response option on the ease of use questions from the device usage questionnaire assessing ease of use (A) and confidence (B) before (previous nebulizer) and after [I-neb Adaptive Aerosol Delivery (AAD) System] the study.

into the lungs, and overall desirability $(p \leq 0.0001$; Table 3 and Fig. 4).

\section{Quality of life}

Quality of life was significantly greater with the I-neb AAD System compared with the patients' previous nebulizer systems based on the CRQ dimensions of dyspnea $(p<0.0001)$ and fatigue $(p=0.015)$ (Fig. 5). The difference between the pre- and poststudy CRQ scores were only clinically meaningful (i.e., difference of $\geq 0.5$ ) for dyspnea. Mean CRQ scores on the dimensions of emotional function and mastery were not significantly different between the preand poststudy CRQ.

\section{Safety}

A total of 75 adverse events were reported by 49 patients during the study. The most common adverse events (reported in equal to or more than two patients) that were not considered device related were chest infection $(n=18)$, exacerbation of COPD $(n=13)$, cough $(n=2)$, shortness of breath $(n=2)$, cold $(n=2)$, and influenza $(n=2)$. Five adverse events were considered device related: sore tongue $(n=1)$, 


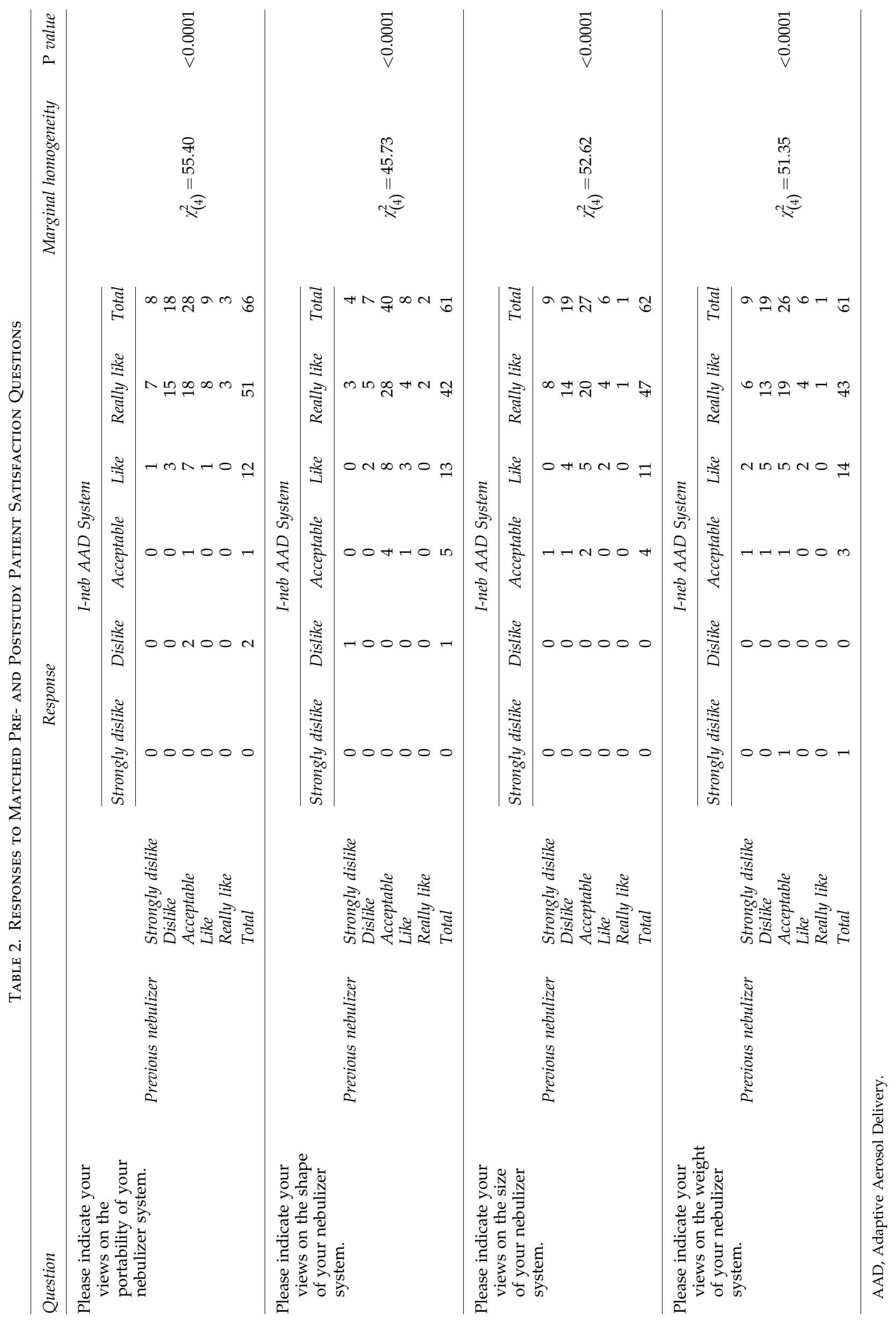




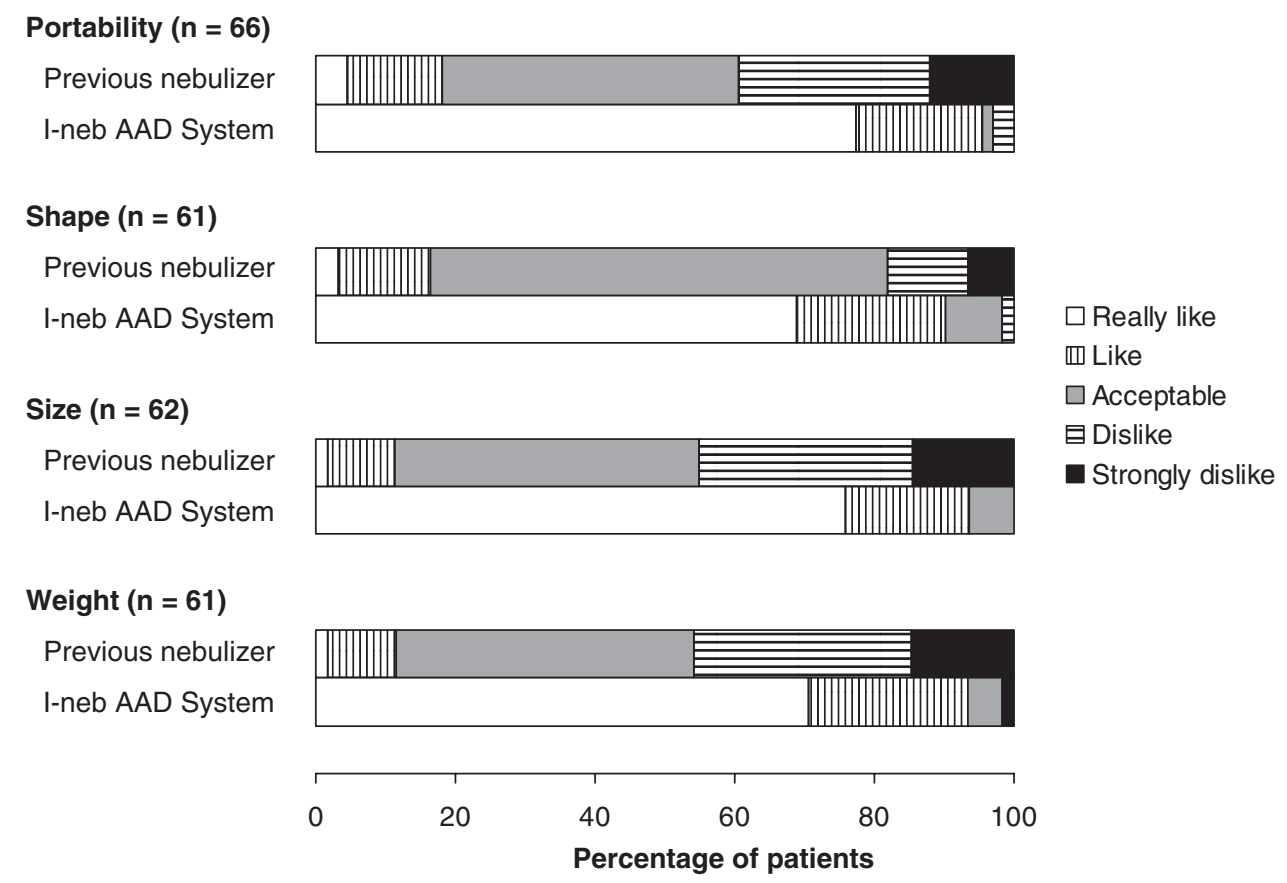

FIG. 3. Percentage of patients reporting each response option on the patient satisfaction questions from the device usage questionnaire before (previous nebulizer) and after [I-neb Adaptive Aerosol Delivery (AAD) System] the study.

headache and nausea $(n=1)$, headache $(n=2)$, and headache and stiff neck $(n=1)$. Sixteen serious adverse events were reported during the study, seven of which resulted in patient withdrawal from the study.

\section{Discussion}

In the present study, patients generally were more satisfied with the I-neb AAD System and found it easier to use than their previous nebulizers (jet nebulizers in most cases) after 3 months of use. Patients also improved in the quality of life CRQ dimensions of dyspnea and fatigue with the I-neb AAD System relative to their previous nebulizers. The specific parameters assessed by each of the ease of use and satisfaction questions suggest that the unique design features of the I-neb AAD System, including physical (e.g., size, shape, weight), mechanical (e.g., those related to assembly, disassembly, cleaning, and operation), and functional (e.g., drug delivery to the lungs) features, might be beneficial to patients with COPD. The results also suggest that patients aged 53-80 years old with COPD could easily transition from using a jet nebulizer to using the I-neb AAD System.

The present results regarding patients' satisfaction with nebulizer treatment are in agreement with previously reported results. ${ }^{(17)}$ A separate group of researchers conducted a survey in the United Kingdom to evaluate patients' opinions of their home nebulizer treatment. ${ }^{(17)}$ Because the survey occurred before the launch of the I-neb AAD System in Europe, it reflects patients' thoughts about their conventional jet or ultrasonic nebulizers. Overall, 76\% (57/75) of respondents had COPD. Approximately $60 \%$ of patients disagreed or strongly disagreed that their home nebulizer treatment "takes up a lot of time during the day" and that their "day revolves around treatment."(17) These findings are consistent with our results, demonstrating that nearly $60 \%$ of patients positively rated (score of 4 or 5) how their previous nebulizer fits with their daily routine; this proportion increased to $97 \%$ for the I-neb AAD System. Providing a nebulizer system that patients prefer and are satisfied with is important as it has been suggested that increased patient satisfaction may lead to improved adherence to treatment and better clinical outcomes. $^{(4)}$

Adherence with nebulized therapy is generally poor in adults with COPD or other chronic lung diseases. ${ }^{(12,13)}$ The I-neb AAD System provides patients with feedback on whether they are adhering to their scheduled medication and using the device correctly. This feedback provides patients with greater control over the correct use of their nebulizer and may improve patient adherence by providing a memory aid. (18) Moreover, some evidence suggests that poor adherence to nebulized therapy leads to impairment in quality of life. Results of a study by a group of researchers at Guy's Hospital in London $(n=82)$ showed that $56 \%$ of patients were poorly adherent (took $<70 \%$ of the prescribed dose for regimens of equal to or less than 4 times daily or $<60 \%$ of the prescribed dose for regimens of equal to or more than 5 times daily) to home nebulizer therapy. ${ }^{(12,13)}$ In their study, poor adherence to treatment was correlated $(p \leq 0.05)$ with decreased quality of life as measured by the symptom subscale, impact subscale, and total score on the St. George's Respiratory Questionnaire at the end of their study. ${ }^{(12,13)}$

Finally, the improvements in quality of life observed in the present study also may be explained by improved drug delivery and increased lung deposition with the I-neb AAD System compared with older conventional nebulizer systems. Lung deposition of ${ }^{99} \mathrm{mTc}$ - diethylene triamine pentaacetic acid (DTPA) delivered via two different breathing modes [Tidal Breathing Mode (TBM) and Target Inhalation Mode (TIM)] with the I-neb AAD System previously was evaluated in 12 healthy subjects in a randomized, open-label, crossover 


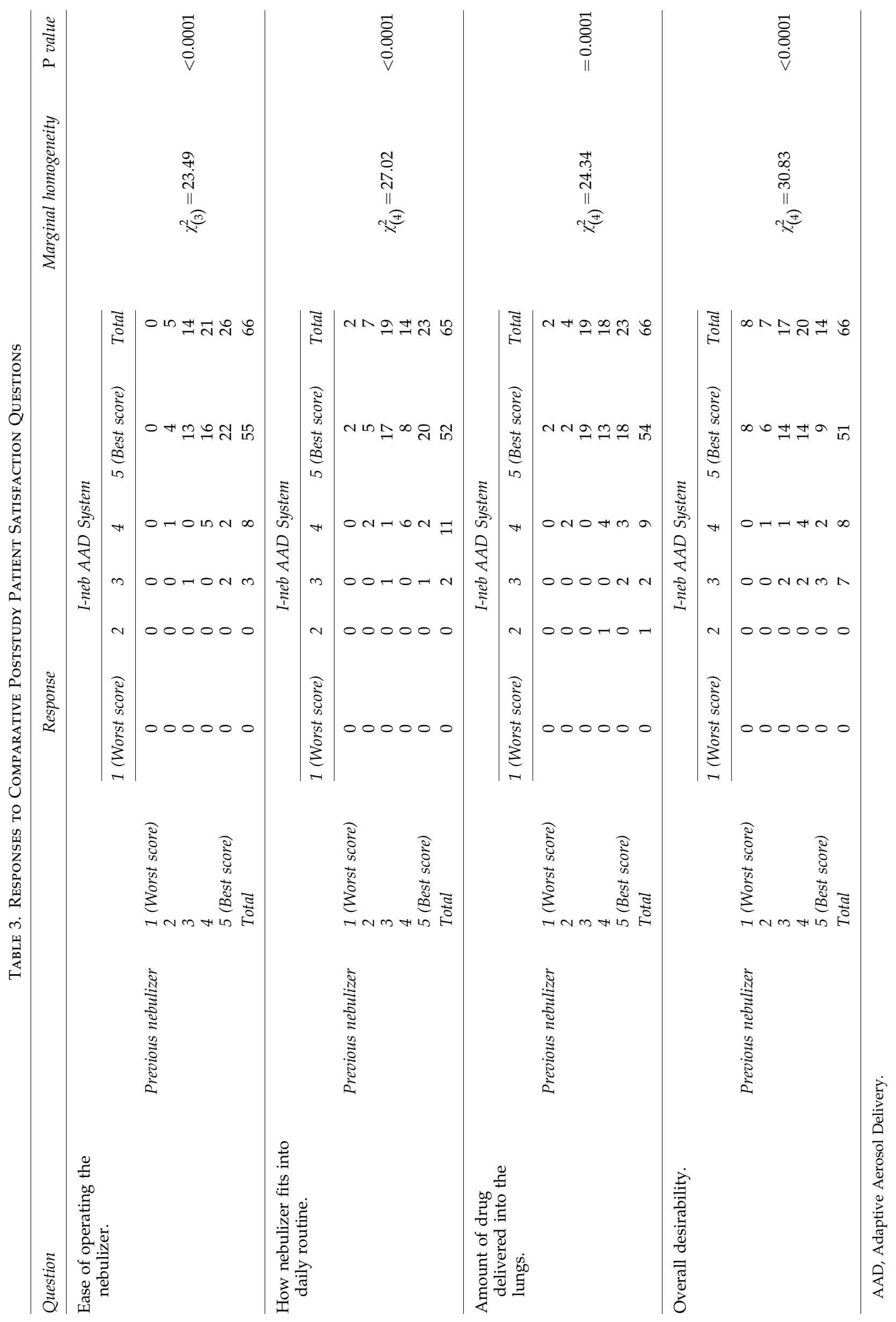




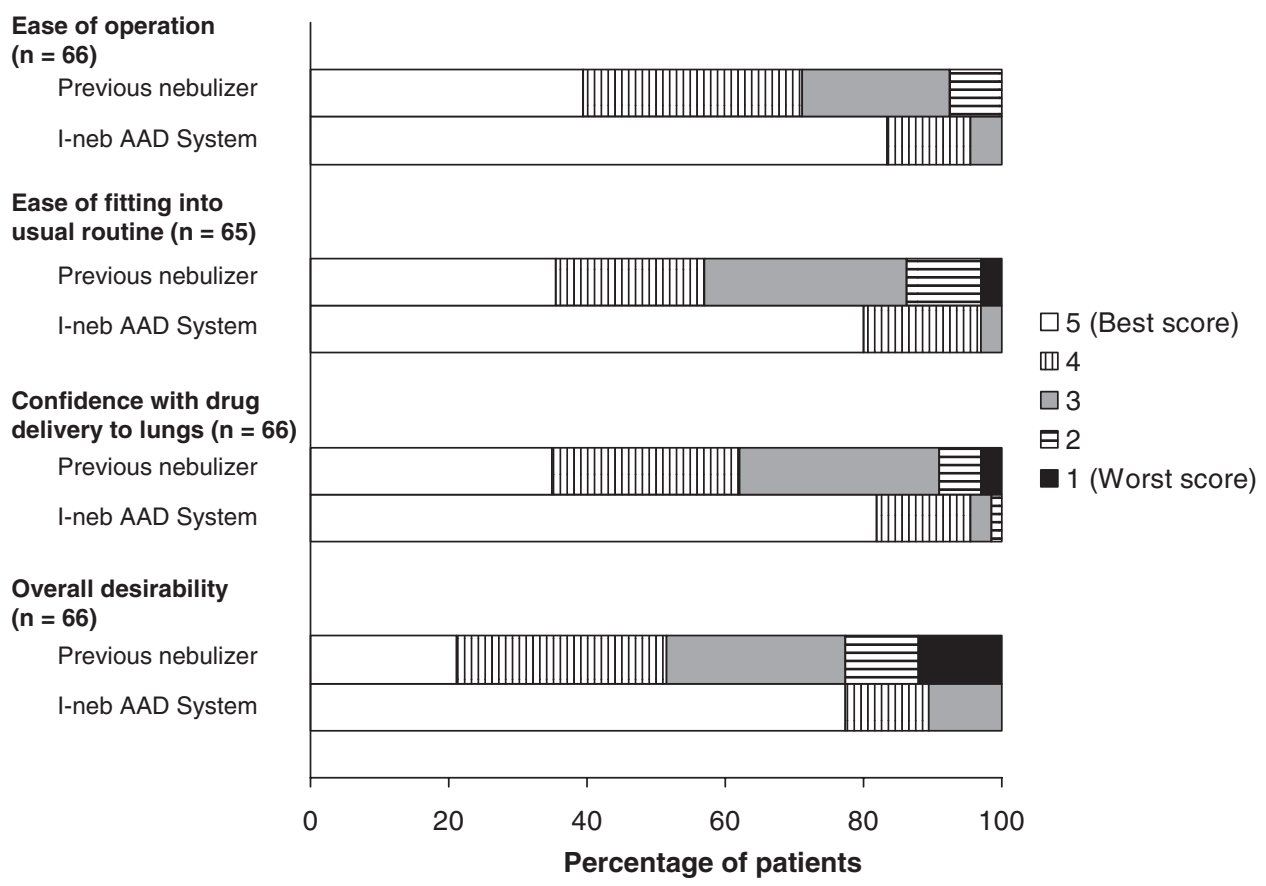

FIG. 4. Percent of patients reporting each response option on the comparative end-of-study satisfaction questions. AAD, Adaptive Aerosol Delivery.

study. ${ }^{(10)}$ The TBM delivered aerosol only during the first half of the patient's inhalation during tidal breathing, whereas TIM guided the patient to perform slow and deep inhalations for about $8 \mathrm{sec}$ and delivered aerosol for up to $7 \mathrm{sec} .{ }^{(10,11)}$ The system provided little wasted medication, with mean exhaled fractions of 1.0 and $0.2 \%$ and mean mouthpiece depositions of 5.3 and $5.0 \%$ with TBM and TIM, respectively. ${ }^{(10)}$ The mean whole lung deposition was 62.8 and $73.3 \%$ when using TBM and TIM, respectively. ${ }^{(10)}$ For comparison, total lung deposition of radiolabeled DTPA administered to 10 healthy subjects and measured with gamma scintigraphy ranged from 2 to $38 \%$ for four different conventional jet nebulizer-compressor systems. ${ }^{(19)}$

Our study has several limitations. Firstly, the patients were comparing their old nebulizer system with the I-neb AAD System in a nonrandomized study design. A randomized study comparing the I-neb AAD System with a specific conventional jet nebulizer-compressor system might have diminished the differences. Nevertheless, the significant differences in the scores on ease of use and satisfaction indicate that the differences in size, shape, and function were of importance. Secondly, the questionnaires covering ease of use and satisfaction were not formally validated. The use of a validated preference questionnaire might have included questions not specifically related to the size, shape, and function of the nebulizers, which probably favored the I-neb AAD System. However, the differences found through the present questionnaire indicate that the patients preferred a small, portable nebulizer. Thirdly, it is possible that participation in the study and preference for the new device may have had positive psychological effects that lead to the improvements in the CRQ dimensions of dyspnea and fatigue. Patients may have expected that their new nebulizer would work better than their old nebulizer, and therefore reported improvements in their dyspnea and fatigue as a result of this expectation. Additionally, the CRQ improvement observed in our study could reflect improved patient adherence to treatment, and/or increased compliance with correct device use.

\section{Conclusions}

In conclusion, the patients with COPD showed significantly greater satisfaction with the I-neb AAD System and found the I-neb AAD System easier to use compared with

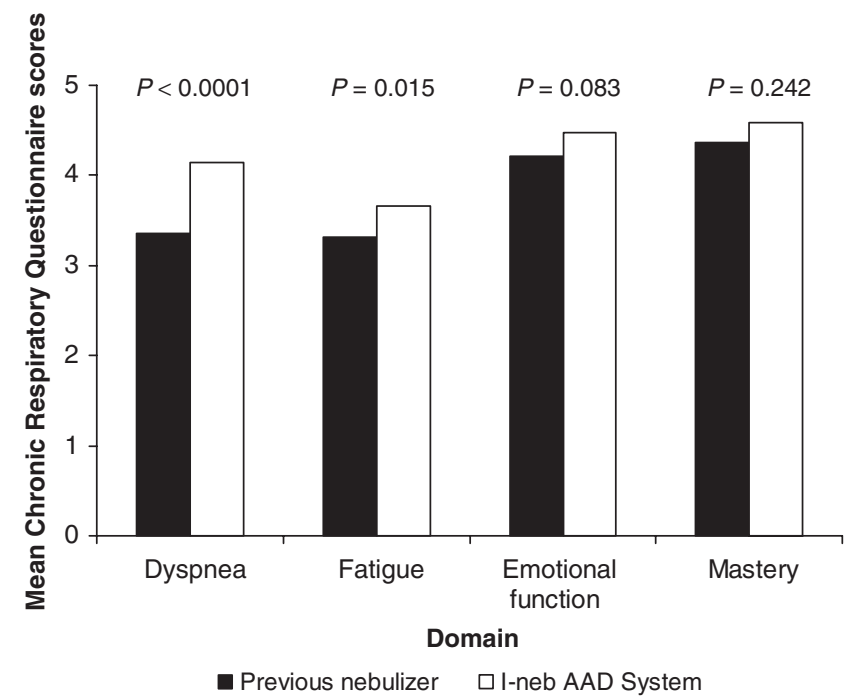

FIG. 5. Mean Chronic Respiratory Questionnaire scores before (previous nebulizer) and after [I-neb Adaptive Aerosol Delivery (AAD) System] the study. 
their previous nebulizers. Patients also had greater confidence that their drug was being delivered to the lungs with the I-neb AAD System compared with their previous nebulizer. Additionally, use of the I-neb AAD System significantly improved dyspnea and fatigue compared with the patient's previous nebulizer system, which may reflect improved adherence or facilitated compliance with the correct use of the I-neb AAD System.

\section{Acknowledgments}

The authors acknowledge N. Smith (PS Writing Ltd, Portsmouth, UK) for his assistance with data analysis and graphics, A. Rigby (Academic Cardiology, University of Hull, Hull, UK) for the statistical analyses, and Scientific Connexions (Newtown, PA) for editorial support. We recognize the inclusion of patients by the following physicians: Dr. A. Jones (Penybryn Surgery, Swansea, UK), Dr. R. Lawson (Royal Hallamshire Hospital, Sheffield, UK), Dr. D.M. Allin (Craig Croft Medical Centre, Birmingham, UK), Dr. M. Parashak (Longford Street Group Practice, Lancashire, UK), Dr. P. Ind (Hammersmith Hospital, London, UK). The study was sponsored by Philips Respironics, Respiratory Drug Delivery (UK) Ltd, Chichester, UK.

\section{Author Disclosure Statement}

The authors declare that no competing financial interests exist. Kurt Nikander, Sarah Hinch, and Steve Coughlin are employees of Philip Respironics.

\section{References}

1. Global Initiative for Chronic Obstructive Lung Disease (GOLD): Global strategy for the diagnosis, management, and prevention of chronic obstructive pulmonary disease. Updated 2007. Available at; http://www.goldcopd.com. Accessed September 30, 2008.

2. Jones PW, Willits LR, Burge PS, and Calverley PM: Inhaled Steroids in Obstructive Lung Disease in Europe study investigators. Disease severity and the effect of fluticasone propionate on chronic obstructive pulmonary disease exacerbations. Eur Respir J. 2003;21:68-73.

3. Dolovich MB, Ahrens RC, Hess DR, Anderson P, Dhand R, Rau JL, Smaldone GC, and Guyatt G: Device selection and outcomes of aerosol therapy: evidence-based guidelines: American College of Chest Physicians/American College of Asthma, Allergy, and Immunology. Chest. 2005;127:335-371.

4. Anderson P: Patient preference for and satisfaction with inhaler devices. Eur Respir Rev. 2005;96:109-116.

5. O'Callaghan C, and Barry PW: The science of nebulized drug delivery. Thorax 1997;52(Suppl 2):S31-S44.

6. Dhand R: Nebulizers that use a vibrating mesh or plate with multiple apertures to generate aerosol. Respir Care. 2002;47: 1406-1416.
7. Nikander K: Drug delivery systems. J Aerosol Med. 1994;7(Suppl 1):S19-S24.

8. Nikander K, Turpeinen $\mathrm{M}$, and Wollmer P: The conventional ultrasonic nebulizer proved inefficient in nebulizing a suspension. J Aerosol Med. 1999;12:47-53.

9. Steckel H, and Eskandar F: Factors affecting aerosol performance during nebulization with jet and ultrasonic nebulizers. Eur J Pharm Sci. 2003;19:443-455.

10. Nikander $\mathrm{K}$, and Denyer J: Adaptive Aerosol Delivery $\left(\mathrm{AAD}^{\circledR}\right)$ Technology. In: Rathbone MJ (ed). Modified Release Drug Delivery Technology, 2nd ed. Informa Healthcare USA, Inc., New York; pp. 603-612, 2008.

11. Waldrep JC, and Dhand R: Advanced nebulizer designs employing vibrating mesh/aperture plate technologies for aerosol generation. Curr Drug Deliv. 2008;5:114-119.

12. Bosley CM, Corden ZM, Rees PJ, and Cochrane GM: Psychological factors associated with use of home nebulized therapy for COPD. Eur Respir J. 1996;9:2346-2350.

13. Corden ZM, Bosley CM, Rees PJ, and Cochrane GM: Home nebulized therapy for patients with COPD: patient compliance with treatment and its relation to quality of life. Chest. 1997;112;1278-1282.

14. NICE guidelines: Diagnosing COPD. Thorax. 2004;59;i27i38.

15. Guyatt GH, Townsend M, Berman LB, and Pugsley SO: Quality of life in patients with chronic airflow limitation. Br J Dis Chest. 1987;81:45-54.

16. Schünemann HJ, Puhan $M$, Goldstein R, Jaeschke R, and Guyatt GH: Measurement properties and interpretability of the chronic respiratory disease questionnaire (CRQ). COPD. 2005;2:81-89.

17. Barta SK, Crawford A, and Roberts CM: Survey of patients' views of domiciliary nebulizer treatment for chronic lung disease. Respir Med. 2002;96:375-381.

18. Chapman KR, Walker L, Cluley S, and Fabbri L: Improving patient compliance with asthma therapy. Respir Med. 2000;94:2-9.

19. Hardy JG, Newman SP, and Knoch M: Lung deposition from four nebulizers. Respir Med. 1993;87:461-465.

Received on June 3, 2009 in final form, November 29, 2009

Reviewed by: Paula Anderson

Address correspondence to: Kurt Nikander, B.A. Philips Respironics Respiratory Drug Delivery 5 Wood Hollow Road Parsippany, NJ 07054

E-mail: Kurt.nikander@philips.com 